\title{
Resource Allocation in Relay-Aided OFDM Cognitive Radio Networks
}

\author{
Guftaar Ahmad Sardar Sidhu, Feifei Gao, Member, IEEE, Wei Wang, and Wen Chen, Senior Member, IEEE
}

\begin{abstract}
In this paper, we investigate the resource allocation problem in a single-user relay-aided cognitive radio (CR) underlay network. Both the CR network and the primary network operate under the orthogonal frequency-division multiplexing (OFDM) scheme. Different from the conventional resource allocation problem, the relay node here is capable of performing subcarrier permutation over two hops such that the signal received over a particular subcarrier is forwarded via a different subcarrier. The objective is to maximize the throughput of the CR network subject to a limited power budget at the secondary source and the relay node and to interference constraints at the primary receiver. Optimization is performed under a unified framework where power allocation at the source node, power allocation at the relay node, and subcarrier pairing at the two hops are jointly optimized. The joint resource-allocation scheme yields an asymptotically optimal solution. We further design a suboptimal algorithm that sacrifices little on performance but could significantly reduce computational complexity. Finally, numerical examples are provided to corroborate the proposed studies.
\end{abstract}

Index Terms-Cognitive radio (CR), orthogonal frequencydivision multiplexing (OFDM), relay network, resource allocation, subcarrier pairing.

\section{INTRODUCTION}

C OGNITIVE radio (CR) is a promising solution to the problem of spectrum scarcity [1], [2], in which an unlicensed secondary user ( $\mathrm{SU}$ ) is allowed to opportunistically access the spectrum of a licensed primary user (PU) when the latter is idle or to share the spectrum simultaneously subject to certain interference limitations [3]-[5]. In the meantime, orthogonal frequency-division multiplexing (OFDM) has shown

Manuscript received September 19, 2011; revised July 26, 2012 and December 17, 2012; accepted March 29, 2013. Date of publication April 23, 2013; date of current version October 12, 2013. This work was supported in part by the National Basic Research Program of China (973 Program) under Grant 2013CB336600 and Grant 2012CB316102; by the National Natural Science Foundation of China under Grant 61201187; by the Scientific Research Foundation for the Returned Overseas Chinese Scholars, State Education Ministry; and by the Tsinghua University Initiative Scientific Research Program under Grant 20121088074. The review of this paper was coordinated by Prof. B. Hamdaoui.

G. A. S. Sidhu was with the School of Engineering and Science, Jacobs University, Bremen 28759, Germany. He is now with the Department of Electrical Engineering, COMSATS Institute of Information Technology, Islamabad, Pakistan, and also with the State Key Laboratory of Integrated Services Networks, Xidian University, Xi'an 710126, China (e-mail: guftaarahmad@comsats.edu.pk).

F. Gao is with Tsinghua National Laboratory for Information Science and Technology, Tsinghua University, Beijing 100084, China, and also with the School of Engineering and Science, Jacobs University, Bremen 28759, Germany (e-mail: feifeigao@ieee.org).

W. Wang is with the Department of Electrical Engineering and Computer Science, South Dakota State University, Brookings, SD 57007 USA (e-mail: wei.wang@sdstate.edu).

W. Chen is with the Department of Electronics Engineering, Shanghai Jiao Tong University, Shanghai 200240, China (e-mail: wenchen@ @jtu.edu.cn).

Digital Object Identifier 10.1109/TVT.2013.2259511 its great potential to combat intersymbol interference and enhance transmission efficiency [6]. Thus, the combination of OFDM and CR becomes an important technology for futuregeneration wireless systems [7]-[15].

In [7], the power allocation problem in OFDM-based CR networks under the overlay transmission protocol was studied. This work was later extended to the relay-aided transmission scenario in [8], where a suboptimal algorithm that optimizes source and relay power allocation in an alternating fashion was proposed. However, neither [7] nor [8] considered the battery limitations of transmitting nodes, i.e., maximum power constraints are missing. The problem of throughput maximization in multicarrier CR systems under transmit power and interference constraints for the underlay $\mathrm{CR}$ scheme was investigated in [9] and was later extended to an amplify-and-forward (AF)relay-aided transmission in [10]. Although the study in [9] is based on the sum power constraint, a more natural choice should be the individual battery power constraint for each transmitting node. Cooperative relaying in OFDM-based CR networks was explored in [11], where the optimal strategy of spectrum allocation and relay assignment was proposed. Recently, a joint algorithm for spectrum sensing adaptation and resource allocation optimization has been proposed in [12]. Furthermore, power allocation in multiple-relay scenarios, relay selection schemes under cooperative transmission, and optimal route selection in multihop CR networks were studied in [13]-[15], respectively.

In a multihop network, the channel gains over different hops are mutually independent. Hence, the subcarriers that experience deep fading over one hop may not experience deep fading over the other hops. This brings a new degree of freedom in resource allocation, which is to properly match the subcarrier of different hops, also known as subcarrier pairing [16]-[18]. The concept of subcarrier pairing was first introduced in [16], where Herdin showed that the system throughput can be enhanced if the subcarriers on the two hops are coupled in the order of their channel magnitude. ${ }^{1}$ Later, in [17] and [18], joint power allocation and the subcarrier pairing problem in $\mathrm{AF}$ and decode-andforward relay networks, respectively, were considered, whereas that in the multiuser two-way relay scenario was exploited in [19]. In CR networks, however, subcarrier pairing faces a new challenge and is a nontrivial task. For example, a subcarrier with the highest channel gain over the first hop may also have the strongest interference to the PUs, and pairing this subcarrier with the strongest subcarrier over the second hop may result in strong interference with the PU. Thus, a more considerate

\footnotetext{
${ }^{1}$ An equal power-allocation strategy is adopted at the source and relay nodes.
} 


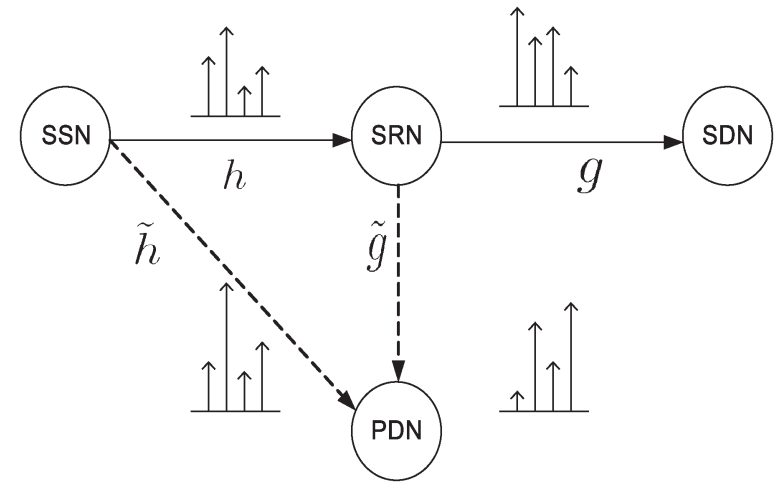

Fig. 1. Relay-enhanced CR network model.

subcarrier pairing strategy is required, i.e., jointly embracing the throughput maximization of the SU and the interference mitigation to the PU. To the best of the authors' knowledge, subcarrier pairing in AF-relay-enhanced CR networks has yet to be explored.

In this paper, we consider an AF-relay-aided CR network that is operated with the OFDM scheme and the underlay spectrum sharing strategy. We seek for optimal resource allocation at the source and the relay, including power allocation over different subcarriers at secondary source/relay nodes and subcarrier pairing at the secondary relay station. We also propose a suboptimal low-complexity algorithm to trade performance for computational complexity. The simulation results show that the suboptimal scheme yields very close performance to the joint optimization algorithm.

The remainder of this paper is organized as follows: In Section II, we present the system model of the relay-aided CR transmission and formulate the joint resource allocation problem. In Section III, we develop a joint optimization algorithm from the dual composition method. In Section IV, we design a low-complexity resource-allocation scheme. Numerical results are presented in Section V to corroborate the proposed studies, and conclusions are made in Section VI.

\section{System Model and Problem Statement}

\section{A. System Model}

Consider a relay-aided CR network where a secondary source node (SSN) communicates with a secondary destination node (SDN) with the help of a secondary relay node (SRN), as shown in Fig. 1. Underlay CR transmission is adopted where the unlicensed SU simultaneously shares the whole spectrum with the licensed PU. Similar to [7]-[15], we assume that the instantaneous channel coefficients for all links are known a prior while such knowledge can be obtained from channel estimation techniques [20], [21] and feedback approaches [22], [23]. More practical considerations, e.g., using statistical channel knowledge or the erroneous channel knowledge [24], are out of the scope of this work. The SSN and SDN are located far from each other such that the direct communication link is missing [17]. On the other hand, the primary destination node (PDN) is located close enough to both the SSN and the SRN such that it experiences interference during both transmission phases (see Fig. 1). OFDM transmission with $K$ subcarriers is applied for the CR network. Unlike the work in [10], where Shamim et al. assumed that the signal received at the SRN over a particular subcarrier is forwarded to the destination over the same subcarrier, we adopt a tone permutation policy such that the signal received on subcarrier $k$ over the SSN-to-SRN link is forwarded via subcarrier $j$ over the SRN-to-SDN link, where $j$ is not necessarily equal to $k$.

For the SSN-to-SRN link, we denote the channel coefficient over the $k$ th subcarrier as $h_{k}$, the power allocation as $p_{k}$, and the transmitted symbol as $x_{k}$. The signal received at the SRN over the $k$ th subcarrier is

$$
y_{k}^{\mathrm{SRN}}=\sqrt{p_{k}} h_{k} x_{k}+z_{k}
$$

where $z_{k}$ is additive white Gaussian noise (AWGN) with variance $\sigma_{k}^{2}$. The signal received over the $j$ th subcarrier at the SDN is then

$$
y_{j}^{\mathrm{SDN}}=\sqrt{q_{j}} \eta_{k}\left(g_{j} h_{k} \sqrt{p_{k}} x_{k}+g_{j} z_{k}\right)+w_{j}
$$

where $\eta_{k} \triangleq\left(\sqrt{p_{k}\left|h_{k}\right|^{2}+\sigma_{k}^{2}}\right)^{-1}, q_{j}$ is the transmit power allocated to the $j$ th subcarrier, $g_{j}$ denotes the corresponding channel coefficient between the SRN and the SDN, and $w_{j}$ represents AWGN at the SDN with variance $\sigma_{j}^{2}$.

The throughput over subcarrier pair $(k, j)$ at a high signal-tonoise ratio (SNR) can be approximated by [17], [25]

$$
c_{(k, j)} \approx \frac{1}{2} \log _{2}\left(1+\frac{\left(\sqrt{p_{k}}\left|h_{k}\right| \sqrt{q_{j}}\left|g_{j}\right|\right)^{2}}{\sigma_{j}^{2}\left(\sqrt{p_{k}}\left|h_{k}\right|\right)^{2}+\sigma_{k}^{2}\left(\sqrt{q_{j}}\left|g_{j}\right|\right)^{2}}\right)
$$

where factor $1 / 2$ is due to the two time slots used in a complete transmission. In particular, [25] demonstrated that the resource allocation obtained from (3) yields very close results to the actual throughput even in the low-SNR scenario.

\section{B. Constraint Definitions}

Since each subcarrier over hop-1 can be paired with one and only one subcarrier in hop-2, we define $\pi_{(k, j)} \in\{0,1\}$ as the binary variable such that

$$
\pi_{(k, j)}= \begin{cases}1, & \text { if the } k \text { th subcarrier of the first hop is paired } \\ \text { with the } j \text { th subcarrier of the second hop } \\ 0, & \text { otherwise. }\end{cases}
$$

With the given definition, the subcarrier pairing constraint can be mathematically expressed as

$$
\sum_{k=1}^{K} \pi_{(k, j)}=1, \quad \forall j \quad \sum_{j=1}^{K} \pi_{(k, j)}=1, \quad \forall k .
$$

Under the proposed transmission protocol, the SSN and the SRN cause interference to the PDN in the first and second time slots, respectively. To protect the PDN from excessive interference, power allocation at the SSN and the SRN must satisfy

$$
\sum_{k=1}^{K} p_{k}\left|\tilde{h}_{k}\right|^{2} \leq I_{\mathrm{th}}, \quad \sum_{j=1}^{K} q_{j}\left|\tilde{g}_{j}\right|^{2} \leq I_{\mathrm{th}}
$$


where $\tilde{h}_{k}$ and $\tilde{g}_{j}$ denote the channel coefficients of SSN-toPDN and SRN-to-PDN links, respectively, whereas $I_{\text {th }}$ denotes the maximum allowable interference threshold. ${ }^{2}$ If the $\mathrm{PU}$ is more sensitive to interference, a per-subcarrier-based interference constraint can be imposed as

$$
p_{k}\left|\tilde{h}_{k}\right|^{2} \leq I_{\mathrm{th}}^{k}, \quad \forall k \quad q_{j}\left|\tilde{g}_{j}\right|^{2} \leq I_{\mathrm{th}}^{j}, \quad \forall j .
$$

Following [17], we assume two types of power constraints:

I) separate transmit power constraints at the SSN and the SRN such that the summation of power allocated over all subcarriers is within the maximum available power budget of the corresponding node, i.e.,

$$
\sum_{k=1}^{K} p_{k} \leq P_{\mathrm{SSN}}, \quad \sum_{j=1}^{K} q_{j} \leq P_{\mathrm{SRN}} ;
$$

II) a sum power constraint over the SSN and the SRN, i.e.,

$$
\sum_{k=1}^{K} p_{k}+\sum_{j=1}^{K} q_{j} \leq P_{T}
$$

\section{Problem Formulation}

Our target is to jointly optimize power allocation at the SSN, power allocation at the SRN, and subcarrier pairing at the two hops such that the overall system throughput of the $\mathrm{SU}$ is maximized subject to the maximum power constraints of the battery and the allowable interference constraints of the PU.

Remark: The difference from the power allocation used in a conventional relay network is illustrated here. In conventional relay networks, a better power-allocation scheme is to set $p_{k}>$ $p_{k^{\prime}}$ and $q_{j}>q_{j^{\prime}}$ whenever $\left|h_{k}\right|^{2}>\left|h_{k^{\prime}}\right|^{2}$ and $\left|g_{j}\right|^{2}>\left|g_{j^{\prime}}\right|^{2}{ }^{3}$. However, in CR-based relay networks, for $\left|h_{k}\right|^{2}>\left|h_{k^{\prime}}\right|^{2}$ and $\left|g_{j}\right|^{2}>\left|g_{j^{\prime}}\right|^{2}$ but $\left|\tilde{h}_{k}\right|^{2}>\left|\tilde{h}_{k^{\prime}}\right|^{2}$ and $\left|\tilde{g}_{j}\right|^{2}>\left|\tilde{g}_{j^{\prime}}\right|^{2}$, we may still need $p_{k}<p_{k^{\prime}}$ and $q_{j}<q_{j^{\prime}}$.

Mathematically, we should optimize over variables $\pi=$ $\left\{\pi_{(k, j)} \in\{0,1\}\right\}, \mathbf{p}=\left\{p_{k} \geq 0\right\}$, and $\mathbf{q}=\left\{q_{j} \geq 0\right\}$ for all $k=\{1, \ldots, K\}, j=\{1, \ldots, K\}$. The following four problems are formulated:

P1: optimization under the separate power constraint and the sum interference constraint, i.e.,

$$
\max _{\boldsymbol{\pi}, \mathbf{p}, \mathbf{q}}, \quad \sum_{k=1}^{K} \sum_{j=1}^{K} \pi_{(k, j)} c_{(k, j)}, \quad \text { s.t. } \quad(4),(5),(7) ;
$$

P2: optimization under the separate power constraint and the per-subcarrier interference constraint, i.e.,

$$
\max _{\boldsymbol{\pi}, \mathbf{p}, \mathbf{q}} \sum_{k=1}^{K} \sum_{j=1}^{K} \pi_{(k, j)} c_{(k, j)}, \quad \text { s.t. } \quad(4),(6),(7) \text {; }
$$

\footnotetext{
${ }^{2}$ The interference threshold is adopted based on quality-of-service requirements of primary users.

${ }^{3}$ More power is allocated to the stronger subcarriers while less power is allocated to the weaker subcarriers.
}

P3: optimization under the sum power constraint and the sum interference constraint, i.e.,

$$
\max _{\boldsymbol{\pi}, \mathbf{p}, \mathbf{q}} \sum_{k=1}^{K} \sum_{j=1}^{K} \pi_{(k, j)} c_{(k, j)}, \quad \text { s.t. } \quad(4),(5),(8) ;
$$

P4: optimization under the sum power constraint and the persubcarrier interference constraint, i.e.,

$$
\max _{\pi, \mathbf{p}, \mathbf{q}} \sum_{k=1}^{K} \sum_{j=1}^{K} \pi_{(k, j)} c_{(k, j)}, \quad \text { s.t. } \quad(4),(6),(8) .
$$

In the following, we shall discuss the solution to problem P1 in detail, whereas solutions to P2, P3, and P4 follow similar steps and are summarized in the corresponding sections.

\section{Joint Resource-Allocation Scheme}

Due to subcarrier pairing constraint (4), problem (9) is classified as mixed-integer programming, which is, in general, difficult to solve. Under the time-sharing condition [26], the duality gap of (9) is proved to be asymptotically zero for sufficiently large $K$, and a rigorous investigation can be found in [27]. Since the time-sharing condition is readily satisfied in our case, we can solve the dual problem of (9), which is defined as [28]

$$
\min _{\lambda \geq 0, \mu \geq 0, \tilde{\lambda} \geq 0, \tilde{\mu} \geq 0} D(\lambda, \mu, \tilde{\lambda}, \tilde{\mu})
$$

where $\lambda, \mu, \tilde{\lambda}$, and $\tilde{\mu}$ are dual variables associated with the constraints in (5) and (7), and dual function $D(\lambda, \mu, \tilde{\lambda}, \tilde{\mu})$ is given by

$D(\lambda, \mu, \tilde{\lambda}, \tilde{\mu})=\max _{\mathbf{p}, \mathbf{q}, \boldsymbol{\pi}} L(\mathbf{p}, \mathbf{q}, \boldsymbol{\pi}, \lambda, \mu, \tilde{\lambda}, \tilde{\mu}), \quad$ s.t. $\quad$ (4).

The Lagrangian in (14) is defined as

$$
\begin{aligned}
& L(\mathbf{p}, \mathbf{q}, \boldsymbol{\pi}, \lambda, \mu, \tilde{\lambda}, \tilde{\mu}) \\
& =\sum_{k=1}^{K} \sum_{j=1}^{K} \pi_{(k, j)} c_{(k, j)}+\lambda\left(P_{\mathrm{SSN}}-\sum_{k=1}^{K} p_{k}\right) \\
& \quad+\mu\left(P_{\mathrm{SRN}}-\sum_{j=1}^{K} q_{j}\right)+\tilde{\lambda}\left(I_{\mathrm{th}}-\sum_{k=1}^{K} p_{k}\left|\tilde{h}_{k}\right|^{2}\right) \\
& \quad+\tilde{\mu}\left(I_{\mathrm{th}}-\sum_{j=1}^{K} q_{j}\left|\tilde{g}_{j}\right|^{2}\right) .
\end{aligned}
$$

\section{A. Solving the Dual Function}

To solve the dual problem in (13), we need to first find the solution of (14). A Lagrange dual decomposition approach is used to obtain the optimum dual function for the given dual variables $\lambda, \mu, \tilde{\lambda}$, and $\tilde{\mu}$. 
From subcarrier pairing constraint (4), we have $\sum_{k=1}^{K} d_{k}=$ $\sum_{k=1}^{K} \sum_{j=1}^{K} \pi_{(k, j)} d_{k}, \quad \sum_{j=1}^{K} e_{j}=\sum_{k=1}^{K} \sum_{j=1}^{K} \pi_{(k, j)} e_{j}$, where $d_{k} \in\left\{p_{k}, p_{k}\left|\tilde{h}_{k}\right|^{2}\right\}$ and $e_{j} \in\left\{q_{j}, q_{j}\left|\tilde{g}_{j}\right|^{2}\right\}$. Using these observations, the objective in (14) can be restated as in (16), shown at the bottom of the page.

Thus, (14) is decomposed into the following two subproblems.

1) Subproblem 1: For any valid subcarrier pair $(k, j)$, i.e., $\pi_{k, j}=1$, the optimal power allocation $p_{k}^{*}$ and $q_{j}^{*}$ could be found from

$$
\begin{array}{cl}
\max _{p_{k}, q_{j}} \quad c_{(k, j)}-p_{k}\left(\lambda+\tilde{\lambda}\left|\tilde{h}_{k}\right|^{2}\right)-q_{j}\left(\mu+\tilde{\mu}\left|\tilde{g}_{j}\right|^{2}\right) \\
\text { s.t. } \quad p_{k} \geq 0, q_{j} \geq 0 .
\end{array}
$$

Since (17) is a standard convex problem, the optimal solution can be obtained from the Karush-Kuhn-Tucker (KKT) conditions as

$$
\begin{gathered}
p_{k}^{*}=\frac{1}{\left(1+\sqrt{\frac{\alpha_{(k, j)}}{\beta_{(k, j)}}}\right)}\left(\frac{1}{\lambda+\tilde{\lambda}\left|\tilde{h}_{k}\right|^{2}}-\frac{\left(\sqrt{\alpha_{(k, j)}}+\sqrt{\beta_{(k, j)}}\right)^{2}}{a_{k} \beta_{(k, j)}}\right)^{+} \\
q_{j}^{*}=\frac{1}{\left(1+\sqrt{\frac{\beta_{(k, j)}}{\alpha_{(k, j)}}}\right)}\left(\frac{1}{\mu+\tilde{\mu}\left|\tilde{g}_{j}\right|^{2}}-\frac{\left(\sqrt{\alpha_{(k, j)}}+\sqrt{\beta_{(k, j)}}\right)^{2}}{\alpha_{(k, j)} b_{j}}\right)^{+}
\end{gathered}
$$

where $a_{k} \triangleq\left|h_{k}\right|^{2} / \sigma_{k}^{2}, b_{j} \triangleq\left|g_{j}\right|^{2} / \sigma_{j}^{2}, \alpha_{(k, j)} \triangleq a_{k}\left(\mu+\tilde{\mu}\left|\tilde{g}_{j}\right|^{2}\right)$, $\beta_{(k, j)} \triangleq\left(\lambda+\tilde{\lambda}\left|\tilde{h}_{k}\right|^{2}\right) b_{j}$, and $(v)^{+} \triangleq \max (0, v)$. Detailed derivation steps are provided in the Appendix.

Remark: Different from the conventional water-filling solution where power depends only on the dual variables (water- level) that are associated with the power constraint (i.e., $\lambda, \mu$ ), the water level here also depends on the dual variables that are associated with the PU's interference constraints (i.e., $\tilde{\lambda}, \tilde{\mu}$ ). Further, (18) and (19) show that the power at the $k$ th subcarrier of the first hop and the $j$ th subcarrier of the second hop also depends on the corresponding interference channel (i.e., $\tilde{h}_{k}$ and $\tilde{g}_{j}$, respectively), resulting in a different water level for each subcarrier. Moreover, the power allocation over the $k$ th subcarrier at the SSN also depends on the $j$ th subcarrier's interference channel of the SRN-to-PDN link. Similarly, the power allocation over the $j$ th subcarrier at the SRN also depends on the $k$ th subcarrier's interference channel of the SSN-to-PDN link.

Substituting (18) and (19) into (16), the dual function becomes

$$
\begin{aligned}
D(\lambda, \mu, \tilde{\lambda}, \tilde{\mu})= & \max _{\pi} \sum_{k=1}^{K} \sum_{j=1}^{K} \pi_{(k, j)} F_{(k, j)}(\lambda, \mu, \tilde{\lambda}, \tilde{\mu}) \\
& +\lambda P_{\mathrm{SSN}}+\mu P_{\mathrm{SRN}}+I_{\mathrm{th}}(\tilde{\lambda}+\tilde{\mu})
\end{aligned}
$$

where function $F_{(k, j)}$ is obtained by using the values of $p_{k}^{*}$ and $q_{j}^{*}$ into the objective of (17) and is expressed as (21), shown at the bottom of the page, with $x_{(k, j)} \triangleq\left(\lambda+\tilde{\lambda}\left|\tilde{h}_{k}\right|^{2}\right)\left(\sqrt{\alpha_{(k, j)}}+\right.$ $\left.\sqrt{\beta_{(k, j)}}\right)^{2}$ and $y_{(k, j)} \triangleq\left(\mu+\tilde{\mu}\left|\tilde{g}_{j}\right|^{2}\right)\left(\sqrt{\alpha_{(k, j)}}+\sqrt{\beta_{(k, j)}}\right)^{2}$. Note that $F_{(k, j)}$ is independent of power variables $p_{k}$ and $q_{j}$ and is defined for given values of the dual variables.

2) Subproblem 2: For known power allocation, subcarrier pairing can be obtained from

$$
\max _{\pi} \sum_{k=1}^{K} \sum_{j=1}^{K} \pi_{(k, j)} F_{(k, j)}(\lambda, \mu, \tilde{\lambda}, \tilde{\mu})
$$

s.t. (4).

$$
\begin{aligned}
D(\lambda, \mu, \tilde{\lambda}, \tilde{\mu})= & \max _{\mathbf{p}, \mathbf{q}, \boldsymbol{\pi}} \sum_{k=1}^{K} \sum_{j=1}^{K} \pi_{(k, j)}\left(c_{(k, j)}-p_{k}\left(\lambda+\tilde{\lambda}\left|\tilde{h}_{k}\right|^{2}\right)-q_{j}\left(\mu+\tilde{\mu}\left|\tilde{g}_{j}\right|^{2}\right)\right)+\lambda P_{\mathrm{SSN}}+\mu P_{\mathrm{SRN}}+I_{\mathrm{th}}(\tilde{\lambda}+\tilde{\mu}) \\
= & \max _{\pi}\left\{\sum_{k=1}^{K} \sum_{j=1}^{K} \pi_{(k, j)} \cdot \max _{p_{k}, q_{j}}\left(c_{(k, j)}-p_{k}\left(\lambda+\tilde{\lambda}\left|\tilde{h}_{k}\right|^{2}\right)-q_{j}\left(\mu+\tilde{\mu}\left|\tilde{g}_{j}\right|^{2}\right)\right)\right\} \\
& +\lambda P_{\mathrm{SSN}}+\mu P_{\mathrm{SRN}}+I_{\mathrm{th}}(\tilde{\lambda}+\tilde{\mu})
\end{aligned}
$$

$$
\begin{aligned}
F_{(k, j)}=\log _{2} & \left(\frac{a_{k} b_{j} \alpha_{(k, j)} \beta_{(k, j)}-a_{k} \alpha_{(k, j)} y_{(k, j)}-b_{j} \beta_{(k, j)} x_{(k, j)}+x_{(k, j)} y_{(k, j)}}{\left(1+\sqrt{\frac{\beta_{(k, j)}}{\alpha_{(k, j)}}}\right)\left(\alpha_{(k, j)}^{2} \beta_{(k, j)}-\frac{\alpha_{(k, j)}^{2} x_{(k, j)}}{a_{k}}\right)+\left(1+\sqrt{\frac{\alpha_{(k, j)}}{\beta_{(k, j)}}}\right)\left(\alpha_{(k, j)} \beta_{(k, j)}^{2}-\frac{\beta_{(k, j)}^{2} y_{(k, j)}}{b_{j}}\right)}\right) \\
& -\left(\frac{\left(a_{k} \beta_{(k, j)}-x_{(k, j)}\right)\left(b_{j} \alpha_{(k, j)}+b_{j} \sqrt{\alpha_{(k, j)} \beta_{(k, j)}}\right)+\left(b_{j} \alpha_{(k, j)}-y_{(k, j)}\right)\left(a_{k} \beta_{(k, j)}+a_{k} \sqrt{\alpha_{(k, j)} \beta_{(k, j)}}\right)}{a_{k} b_{j}\left(\beta_{(k, j)}+\sqrt{\alpha_{(k, j)} \beta_{(k, j)}}\right)\left(\alpha_{(k, j)}+\sqrt{\alpha_{(k, j)} \beta_{(k, j)}}\right)}\right)
\end{aligned}
$$


We define a $K \times K$ profit matrix $\boldsymbol{F}$ such that

$$
\boldsymbol{F}=\left[\begin{array}{cccc}
F_{(1,1)} & F_{(1,2)} & \ldots & F_{(1, K)} \\
F_{(2,1)} & F_{(2,2)} & \ldots & F_{(2, K)} \\
: & & : & : \\
\cdot & & \cdot & \cdot \\
F_{(K-1,1)} & F_{(K-1,2)} & \ldots & F_{(K-1, K)} \\
F_{(K, 1)} & F_{(K, 2)} & \ldots & F_{(K, K)}
\end{array}\right]
$$

where the row and column indexes denote different customers and different items to sell, respectively, whereas the value of each entry can be deemed as profit. Problem (22) is equivalent to maximizing the sum profit by an optimal selling strategy that can only sell one item to one customer. This kind of linear assignment problem can be efficiently solved using the standard Hungarian algorithm with complexity $O\left(K^{3}\right)$ [29] and yields optimum subcarrier pairing $\pi^{*}$.

\section{B. Solving the Dual Problem With Subgradient Method}

Substituting $\mathbf{p}^{*}, \mathbf{q}^{*}$, and $\boldsymbol{\pi}^{*}$ into (15) and then the result back into (14), we obtain the optimal dual function for the given values of the dual variables. The optimal dual variables can be obtained from dual problem (13) using the subgradient method [30]. For any initial values $\lambda^{(0)}, \mu^{(0)}, \tilde{\lambda}^{(0)}$, and $\tilde{\mu}^{(0)}$, the dual variables at the $(i+1)$ th iteration could be updated as

$$
\begin{aligned}
& \lambda^{(i+1)}=\left(\lambda^{(i)}-\delta^{(i)} \Delta^{(i)}(\lambda)\right)^{+} \\
& \mu^{(i+1)}=\left(\mu^{(i)}-\delta^{(i)} \Delta^{(i)}(\mu)\right)^{+} \\
& \tilde{\lambda}^{(i+1)}=\left(\tilde{\lambda}^{(i)}-\delta^{(i)} \Delta^{(i)}(\tilde{\lambda})\right)^{+} \\
& \tilde{\mu}^{(i+1)}=\left(\tilde{\mu}^{(i)}-\delta^{(i)} \Delta^{(i)}(\tilde{\mu})\right)^{+}
\end{aligned}
$$

where $\delta^{(i)}$ is the appropriate step size of the $i$ th iteration. Moreover, $\Delta(\lambda), \Delta(\mu), \Delta(\tilde{\lambda})$, and $\Delta(\tilde{\mu})$ are the subgradients of $D(\lambda, \mu, \tilde{\lambda}, \tilde{\mu})$ whose expressions are given by

$$
\begin{aligned}
& \Delta(\lambda)=P_{\mathrm{SSN}}-\sum_{k=1}^{K} p_{k}^{*} \quad \Delta(\mu)=P_{\mathrm{SRN}}-\sum_{j=1}^{K} q_{j}^{*} \\
& \Delta(\tilde{\lambda})=I_{\mathrm{th}}-\sum_{k=1}^{K}\left|\tilde{h}_{k}\right|^{2} p_{k}^{*} \quad \Delta(\tilde{\mu})=I_{\mathrm{th}}-\sum_{j=1}^{K}\left|\tilde{g}_{j}\right|^{2} q_{j}^{*}
\end{aligned}
$$

where $p_{k}^{*}$ and $q_{j}^{*}$ are the optimal power values obtained from (18) and (19) at $\lambda, \mu, \tilde{\lambda}$, and $\tilde{\mu}^{4}{ }^{4}$

Remark: The updates of the subgradients follow an interesting supply and demand strategy. For example, in $\Delta(\lambda)$, $\lambda$ can be considered as the price for power at the source node. The price will be increased if the demand (the total allocated power) is higher than the available stock (the maximum available power). If the demand is less than the available power budget, the price is decreased to encourage the SSN to increase its transmit power.

\footnotetext{
${ }^{4}$ Note that for each iteration, all variables $\pi^{*}, p_{k}^{*}$, and $q_{j}^{*}$ should be recomputed under $\lambda^{(i)}, \mu^{(i)}, \tilde{\lambda}^{(i)}$, and $\tilde{\mu}^{(i)}$. The iterations stop once a certain criterion is fulfilled.
}

\section{Solving Problems P2, P3, and P4}

Optimization problems (10)-(12) can be solved with similar steps as those in Section III-A and B. Some differences are described here.

In $\mathrm{P} 2$, there are $2(K+1)$ dual variables to be updated, i.e.,

$$
\begin{aligned}
& \tilde{\lambda}_{k}^{(i+1)}=\left(\tilde{\lambda}_{k}^{(i)}-\delta^{(i)} \Delta^{(i)}\left(\tilde{\lambda}_{k}\right)\right)^{+} \\
& \tilde{\mu}_{j}^{(i+1)}=\left(\tilde{\mu}_{j}^{(i)}-\delta^{(i)} \Delta^{(i)}\left(\tilde{\mu}_{j}\right)\right)^{+}
\end{aligned}
$$

where $\Delta\left(\tilde{\lambda}_{k}\right) \triangleq I_{\mathrm{th}}^{k}-\left|\tilde{h}_{k}\right|^{2} p_{k}^{*}, \Delta\left(\tilde{\mu}_{j}\right) \triangleq I_{\mathrm{th}}^{j}-\left|\tilde{g}_{j}\right|^{2} q_{j}^{*}$, and $\tilde{\lambda}_{k}$ and $\tilde{\mu}_{j}$ are the dual variables associated with the $k$ th and the $j$ th constraints in (6), respectively. The mathematical expressions for $p_{k}^{*}$ and $q_{j}^{*}$ are similar to those in (18) and (19) and could be obtained by replacing $\tilde{\lambda}$ and $\tilde{\mu}$ with $\tilde{\lambda}_{k}$ and $\tilde{\mu}_{j}$.

In $\mathrm{P} 3$, the separate power constraints in (7) are combined into a single total power constraint. Let $\xi$ be the dual variable associated with the total power constraint in (8). It should be updated from

$$
\xi^{(i+1)}=\left(\xi^{(i)}-\delta^{(i)}\left(P_{T}-\left(\sum_{k=1}^{K} p_{k}^{*}+\sum_{j=1}^{K} q_{j}^{*}\right)\right)\right)^{+}
$$

where $p_{k}^{*}$ and $q_{j}^{*}$ are obtained by substituting $\lambda=\xi$ and $\mu=\xi$ into (18) and (19).

In P4, both (28) and (29) should be taken into consideration.

\section{Suboptimal Algorithm}

The joint subcarrier pairing and power loading scheme in the previous section provides a near-optimal solution when the number of subcarriers is large. However, computational complexity also increases when the number of subcarriers increases. Here, we propose a suboptimal approach that trades performance for lower complexity by separating power allocation from subcarrier pairing.

We solve optimization (9) using a stepwise approach. In the first step of the following algorithm, we find subcarrier pairing for an anticipated power allocation, and then, in the second step, power allocation is done for the obtained pairing.

1) Step 1: It is desirable to allocate more power to good channels (i.e., $h_{k}$ and $g_{k}$ ) to enhance the SU's throughput. Meanwhile, it is also desirable to allocate less power to the strong interference channels $\left(\tilde{h}_{k}\right.$ and $\left.\tilde{g}_{j}\right)$ to reduce interference to PUs. Motivated by these, let us first define the ratio between the actual channel gain to the interference channel gain as

$$
H_{k}=\frac{I_{\mathrm{th}}\left|h_{k}\right|^{2}}{\left|\tilde{h}_{k}\right|^{2}}, \quad \forall k, \quad G_{j}=\frac{I_{\mathrm{th}}\left|g_{j}\right|^{2}}{\left|\tilde{g}_{j}\right|^{2}}, \quad \forall j .
$$

Then, if $H_{k}>H_{k^{\prime}}$, we should set $p_{k}>p_{k^{\prime}}$. Similarly, for $G_{j}>G_{j^{\prime}}$, we should set $q_{j}>q_{j^{\prime}}$. We then propose to pair the carriers in the sorted order of $H_{k}$ and $G_{j}$, i.e., the carrier with the strongest $H_{k}$ is paired with that with the strongest $G_{j}$.

2) Step 2: Next, we seek for power allocation given the subcarrier pairing obtained in step 1. Assume that subcarrier 
$k$ is paired with subcarrier $m(k)$, where $m(\cdot)$ denotes the corresponding mapping function. The optimization in (9) is then

$$
\begin{aligned}
\max _{\mathbf{p}, \mathbf{q}} & \sum_{k=1}^{K} \log _{2}\left(1+\frac{\left(\sqrt{p_{k}}\left|h_{k}\right| \sqrt{q_{m(k)}}\left|g_{m(k)}\right|\right)^{2}}{\sigma_{k}^{2}\left(\sqrt{p_{k}}\left|h_{k}\right|\right)^{2}+\sigma_{m(k)}^{2}\left(\sqrt{q_{m(k)}}\left|g_{m(k)}\right|\right)^{2}}\right) \\
\text { s.t. } & \sum_{k=1}^{K} p_{k}\left|\tilde{h}_{k}\right|^{2} \leq I_{\mathrm{th}}, \sum_{k=1}^{K} q_{m(k)}\left|\tilde{g}_{m(k)}\right|^{2} \leq I_{\mathrm{th}} \\
& \sum_{k=1}^{K} p_{k} \leq P_{\mathrm{SSN}}, \sum_{k=1}^{K} q_{m(k)} \leq P_{\mathrm{SRN}}
\end{aligned}
$$

which can be solved through the dual decomposition technique, similarly as in Section III. Hence, (16) becomes

$$
\begin{aligned}
D(\lambda, \mu, \tilde{\lambda}, \tilde{\mu})=\max _{\mathbf{p}, \mathbf{q}} \sum_{k=1}^{K}\left(\log _{2}\left(1+\operatorname{SNR}_{k}\right)-p_{k}\left(\lambda+\tilde{\lambda}\left|\tilde{h}_{k}\right|^{2}\right)\right. \\
\left.\quad-q_{m(k)}\left(\mu+\tilde{\mu}\left|\tilde{g}_{m(k)}\right|^{2}\right)\right) \\
+\lambda P_{\mathrm{SSN}}+\mu P_{\mathrm{SRN}}+I_{\mathrm{th}}(\tilde{\lambda}+\tilde{\mu})
\end{aligned}
$$

where $\mathrm{SNR}_{k}=\left(\sqrt{p_{k}}\left|h_{k}\right| \sqrt{q_{m(k)}}\left|g_{m(k)}\right|\right)^{2} / \sigma_{k}^{2}\left(\sqrt{p_{k}}\left|h_{k}\right|\right)^{2}+\sigma_{m(k)}^{2}$ $\left(\sqrt{q_{m(k)}}\left|g_{m(k)}\right|\right)^{2}$. The given problem could be decomposed into $K$ subproblems, and then, the KKT conditions can be applied for each. Optimum power variables $p_{k}^{*}$ and $q_{m(k)}^{*}$ could be found from (18) and (19) by replacing $j$ with $m(k)$. Similarly, the dual variable updates are obtained from (24) and (25).

This algorithm could also be extended to P2, P3, and P4. If different subcarriers have different interference requirements, then $H_{k}$ and $G_{j}$ can be redefined as

$$
H_{k}=\frac{I_{\text {th }}^{k}\left|h_{k}\right|^{2}}{\left|\tilde{h}_{k}\right|^{2}}, \quad \forall k, \quad G_{j}=\frac{I_{\text {th }}^{j}\left|g_{j}\right|^{2}}{\left|\tilde{g}_{j}\right|^{2}}, \quad \forall j .
$$

\section{Simulation Results and Discussions}

This section provides numerical examples to demonstrate the performance of the developed schemes. We assume sixtap channels taken from independent and identically distributed Gaussian random variables for all links and choose $K=32$. Without loss of generality, we assume that $P_{\mathrm{SSN}}=P_{\mathrm{SRN}}=$ $P_{\text {max }}$. Further, noise variances at the SRN and the SDN are set as $\sigma_{k}^{2}=\sigma_{j}^{2}, \forall k, j$. The figure of merit is taken as the per-tone rate of the SU, i.e., the sum rate divided by $K$. The value of the step size for subgradient updates is taken as $\delta=1 \times 10^{-3}$. We compare the following four different algorithms.

- JntSol: The joint optimization solution presented in Section III.

- PowSol: The algorithm where only power allocation is performed while subcarrier pairing is not considered. The problem is equivalent to the optimization in (30) with $m(k)=k, \forall k$, and the solution follows the same procedure described in the second step of the suboptimal algorithm.

- LCA: The solution that corresponds to the low-complexity suboptimal algorithm presented in Section IV.
- SUB: A suboptimal algorithm based on the equal-powerallocation scheme developed in [9] for the single-hop system, where, at each node, power is equally distributed among the carriers subject to the condition that the interference constraint is satisfied. Specifically, for our system, the power for $\mathrm{P} 1, \mathrm{P} 2, \mathrm{P} 3$, and $\mathrm{P} 4$ is $v_{i}=\min \left(P_{\max } /\right.$ $\left.K, I_{\mathrm{th}} / \sum_{i=1}^{K} \tilde{f}_{i}\right), v_{i}=\min \left(P_{\max } / K, \min _{i}\left(I_{\mathrm{th}}^{i} / \tilde{f}_{i}\right)\right), v_{i}=$ $\min \left(P_{\max } / 2 K, I_{\mathrm{th}} / \sum_{i=1}^{K} \tilde{f}_{i}\right)$, and $v_{i}=\min \left(P_{\max } / 2 K\right.$ $\left.\min _{i}\left(I_{\mathrm{th}}^{i} / \tilde{f}_{i}\right)\right)$, respectively, with $v_{i} \in\left\{p_{i}, q_{i}\right\}, \tilde{f}_{i} \in\left\{\left|\tilde{h}_{i}\right|^{2}\right.$, $\left.\left|\tilde{g}_{i}\right|^{2}\right\}, i \in\{k, j\}$.

\section{A. Complexity and Convergence}

We first look into the complexities involved in the proposed schemes and then show the convergence behavior of different algorithms.

Algorithm 1: Joint optimization scheme

1) Initialize dual variables and step size.

2) Obtain the power allocation and find $F_{(k, j)}$ for all $k, j$ such that

for $k=1$ to $K$

for $j=1$ to $K$

a) Find $p_{k}^{*}$ and $q_{j}^{*}$ using (18) and (19), respectively.

b) Find $F_{(k, j)}$ from (21)

end for

end for

3) Obtain $\mathbf{F}$ and obtain $\boldsymbol{\pi}^{*}$ from Hungarian method.

4) Update dual variables using (24) and (25).

5) Repeat step 2 to step 4 until convergence

Algorithm 2: Suboptimal scheme

1) Find the subcarrier pairing in the sorted order of $H_{k}$ and $G_{j}$ for all $(k, j)$ and obtain the mapping function $m($. accordingly.

2) Initialize dual variables and step size.

3) Obtain the power allocation such that for $k=1$ to $K$

Find $p_{k}^{*}$ and $q_{j}^{*}=q_{m(k)}$ using (18) and (19), respectively. end for

4) Update dual variables using (24) and (25).

5) Repeat step 3 to step 4 until convergence

The different steps involved in the joint optimization scheme are summarized in Algorithm 1. In step 2, computing $p_{k}$ and $q_{j}$ for all $k, j$ requires a complexity of $O\left(K^{2}\right)$ (multiplications and additions). Furthermore, obtaining $F_{(k, j)}$ involves $O\left(K^{2}\right)$ operations (log, multiplications, additions), where the subcarrier pairing in step 3 is found through the Hungarian algorithm, requiring a complexity of $O\left(K^{3}\right)$. If the objective of the dual function in (13) is minimized in $I$ iterations, the total computational complexity of the joint resource-allocation algorithm in section becomes $O\left(I K^{3}\right)$.

The proposed suboptimal algorithm (LCA) is given in Algorithm 2, where the subcarrier pairing in step 1 involves a 
TABLE I

COMPLEXITY COMPARISON OF DIFFERENT ALGORITHMS

\begin{tabular}{|c|c|cccc|}
\hline Algorithm & Complexity & \multicolumn{4}{|c|}{ Running Time (Seconds) } \\
\cline { 3 - 5 } & & P1 & P2 & P3 & P4 \\
\hline JntSol & $O\left(I K^{3}\right)$ & 366.2310 & 948.64 & 87.1563 & 726.5781 \\
\hline PowSol & $O\left(I^{\prime \prime} K\right)$ & 1.2969 & 3.1563 & 0.0313 & 2.4688 \\
\hline LCA & $O\left(I^{\prime} K\right)$ & 0.4375 & 3.1719 & 0.0156 & 1.5469 \\
\hline SUB & $O(K)$ & 0.0156 & 0.021 & 0.010 & 0.0182 \\
\hline
\end{tabular}

(a)

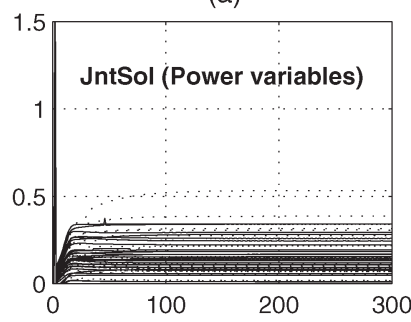

(c)

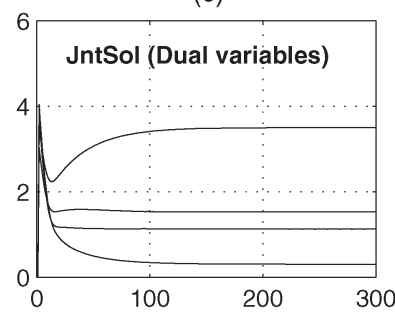

(b)

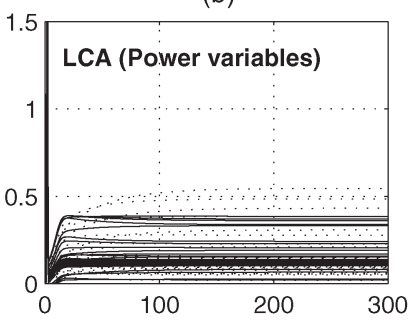

(d)

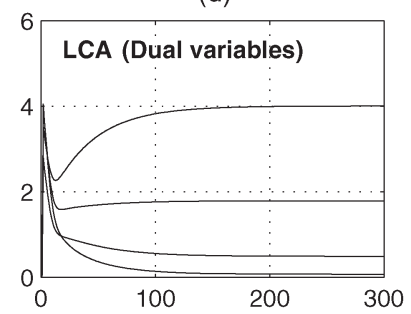

Fig. 2. Convergence for optimization P1. (a) JntSol: primary variables $\left(p_{k}, q_{j}\right)$. (b) LCA: primary variables $\left(p_{k}, q_{j}\right)$. (c) JntSol: dual variables $(\lambda, \mu, \tilde{\lambda}$, and $\tilde{\mu})$. (d) LCA: dual variables $(\lambda, \mu, \tilde{\lambda}$, and $\tilde{\mu})$.

maximum of $2 K$ computations (divisions). Further, the power allocation in step 3 requires $K$ operations (additions, multiplications), and thus, the complexity of solving (30) is $O\left(I^{\prime} K\right)$, where $I^{\prime}$ is the number of iterations required in subgradient search. Without loss of generality, we can set $I^{\prime}$ close to $I$. Then, the overall complexity of the proposed suboptimal algorithm is much less than the complexity of the joint resourceallocation schemes.

Table I summarizes the complexities involved in each algorithm, where $I^{\prime \prime}$ represents the number of iterations needed for the convergence of PowSol. Moreover, the running times of different algorithms for a channel realization at $P_{\max }=5$ reference. ${ }^{5}$ The PU's interference thresholds are set as $=I_{\mathrm{th}}^{k}=$ $I_{\mathrm{th}}^{j}=I_{t} h / K=0.1$.

We look into the convergence behavior of two algorithms proposed in Sections III and IV in Figs. $2-4$, where the $x$-axis represents the number of iterations. Fig. 2 shows the convergence of JntSol and LCA for problem P1. The convergence of the $2 K$ power variables (solid lines are $p_{k}, \forall k$; dashed lines show $\left.q_{j}, \forall j\right)$ and the four dual variables $(\lambda, \mu, \tilde{\lambda}, \tilde{\mu})$ in JntSol are shown in Fig. 2(a) and (c), whereas Fig. 2(b) and (d) shows the convergence of the primal $\left(p_{k}, q_{j}, \forall k, j\right)$ and dual variables $(\lambda, \mu, \tilde{\lambda}, \tilde{\mu})$ for LCA, respectively. The total power at the secondary transmitting nodes is taken as $P_{\max }=5$, and the maximum allowable interference power at the PU is set

\footnotetext{
${ }^{5}$ Although using running time cannot strictly reflect computational complexity, it can still well demonstrate the difference among all the compared algorithms.
}

(a)

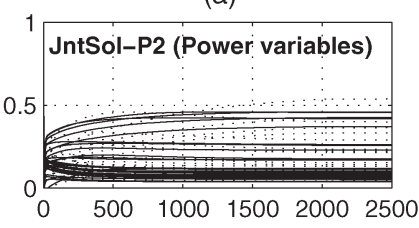

(c)

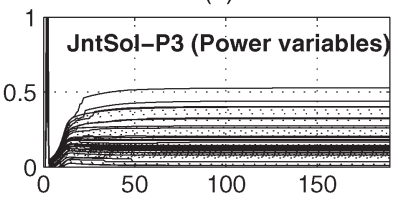

(e)

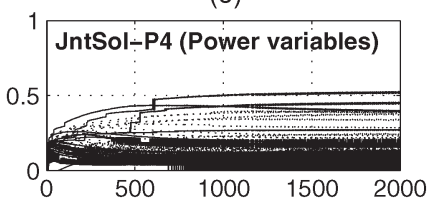

(b)

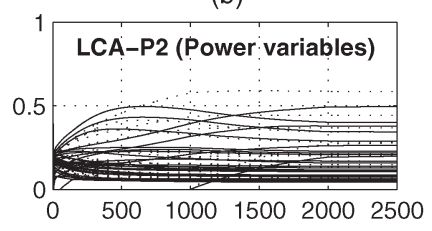

(d)

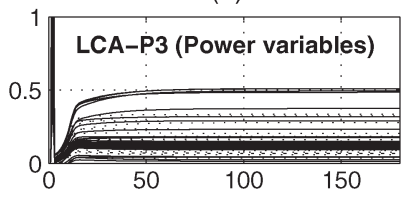

(f)

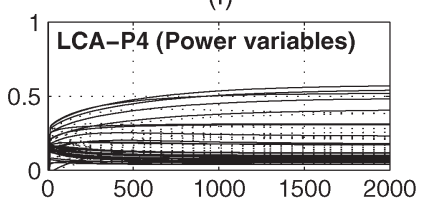

Fig. 3. Convergence behavior of primary variables $\left(p_{k}, q_{j}\right)$ for optimization P2-P4. (a) JntSol: Optimization P2. (b) LCA: Optimization P2. (c) JntSol: Optimization P3. (d) LCA: Optimization P3. (e) JntSol: Optimization P4. (f) LCA: Optimization P4.

(a)

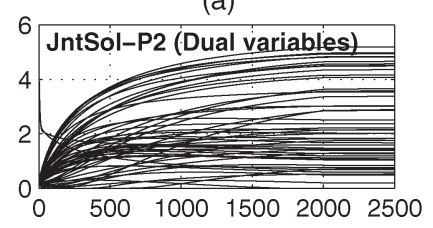

(c)

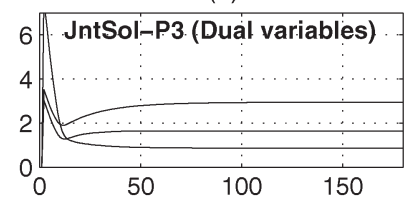

(e)

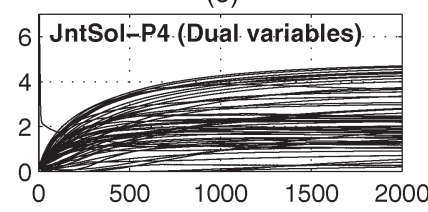

(b)

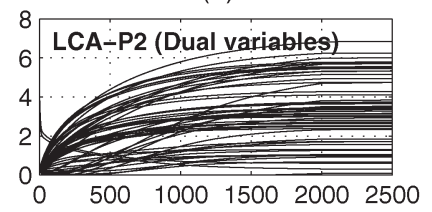

(d)

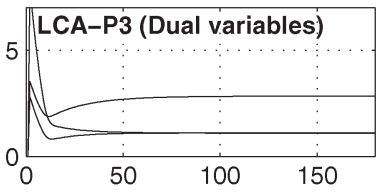

(f)

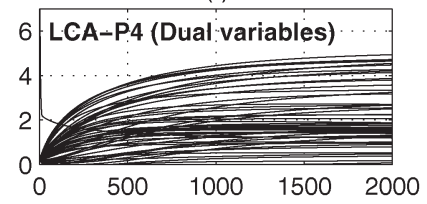

Fig. 4. Convergence behavior of dual variables for optimization P2-P4. (a) JntSol: Optimization P2. (b) LCA: Optimization P2. (c) JntSol: Optimization P3. (d) LCA: Optimization P3. (e) JntSol: Optimization P4. (f) LCA: Optimization P4.

as $I_{\mathrm{th}} / K=0.1$. We observe that as the dual variables converge, the primary variables also converge. Furthermore, both the joint optimal solution and the proposed low-complexity algorithms converge after an almost equal number of iterations, which supports our previous statement that $I$ can be set close to $I^{\prime}$.

Figs. 3 and 4 show the convergence behavior of variables for problems P2, P3, and P4. The different variables plotted in the subplots are summarized in Table II. In Fig. 3, all power variables at the $\operatorname{SSN}\left(p_{k}, \forall k\right)$ are represented with solid blue lines, and all $q_{j}, \forall j$ are shown with dotted red lines. We notice that the problem under the sum power constraint and the sum interference constraint, i.e., P3, requires the least number of 
TABLE II

VARiables Plotted in Figs. 2-4

\begin{tabular}{|c|c|c|c|c|c|c|}
\hline Subplot & a & $\mathrm{b}$ & $\mathrm{c}$ & $\mathrm{d}$ & $\mathrm{e}$ & $\mathrm{f}$ \\
\hline Fig. 2 & JntSol-P1: $p_{k}, q_{j}, \forall k, j$ & LCA-P1: $p_{k}, q_{j}, \forall k, j$ & JntSol-P1: $\lambda, \mu, \tilde{\lambda}, \tilde{\mu}$ & LCA-P1: $\lambda, \mu, \tilde{\lambda}, \tilde{\mu}$ & - & - \\
\hline Fig. 3 & JntSol-P2: $p_{k}, q_{j}, \forall k, j$ & LCA-P2: $p_{k}, q_{j}, \forall k, j$ & JntSol-P3: $p_{k}, q_{j}, \forall k, j$ & LCA-P3: $p_{k}, q_{j}, \forall k, j$ & JntSol-P4: $p_{k}, q_{j}, \forall k, j$ & LCA-P2: $p_{k}, q_{j}, \forall k, j$ \\
\hline Fig. 4 & JntSol-P2: $\lambda, \mu, \tilde{\lambda}_{k}, \forall k, \tilde{\mu}_{j}, \forall j$ & LCA-P2: $\lambda, \mu, \tilde{\lambda}_{k}, \forall k, \tilde{\mu}_{j}, \forall j$ & JntSol-P3: $\lambda, \mu, \xi$ & LCA-P3: $\lambda, \mu, \xi$ & JntSol-P4: $\xi, \tilde{\lambda}_{k}, \forall k, \tilde{\mu}_{j}, \forall j$ & LCA-P2: $\xi, \tilde{\lambda}_{k}, \forall k, \tilde{\mu}_{j}, \forall j$ \\
\hline
\end{tabular}

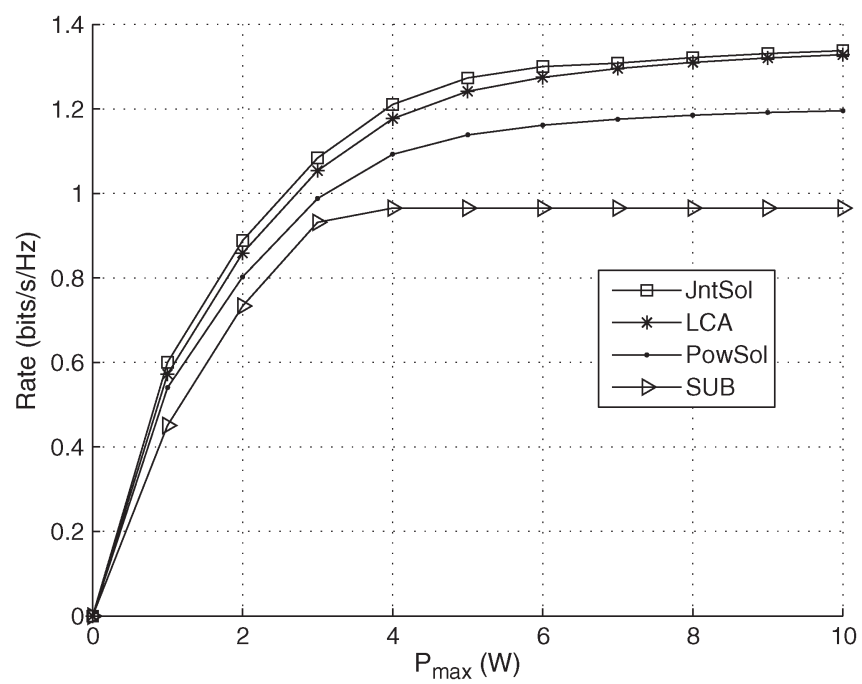

Fig. 5. Rate versus $P_{\max }$. Optimization under the sum interference constraint and the per-node power constraint.

iterations to converge, whereas $\mathrm{P} 2$ requires the utmost number of iterations. Further, we see that in all problems, JntSol and LCA require similar number of iterations. On the other hand, the running time of LCA is much less than that of JntSol in all of the four problems. This is due to the high time complexity of the Hungarian algorithm required in each iteration of JntSol. However, the difference in running times of LCA and PowSol comes from the fast convergence of the LCA, i.e., PowSol requires more iterations to converge than that of LCA.

\section{B. Performance}

Now, we check the performance of the proposed algorithms. In the first example, the performance comparison of four different algorithms under separate power and sum interference constraints is shown in Fig. 5. We display the secondary system's throughput versus $P_{\max }$ at $I_{\mathrm{th}} / K=0.1$. We observe that JntSol exhibits the best performance at all $P_{\max }$ values among all different schemes. Moreover, the proposed suboptimal schemes also provide much better performance over the trivial method (the SUB algorithm). The proposed suboptimal power allocation and subcarrier pairing method, i.e., LCA, achieves large performance gains over the PowSol method and gives very close performance to that of JntSol with much less computational complexity.

Next, we examine the performance of the end-to-end rate under separate power and per-subcarrier interference constraints, i.e., we consider the optimization in (10). The corresponding curves at $I_{\mathrm{th}}^{k}=I_{\mathrm{th}}^{j}=0.1$ are shown in Fig. 6, and to compare with the results in Fig. 5, we set $K I_{\text {th }}^{k}=K I_{\text {th }}^{j}=I_{\text {th }}$. Similar

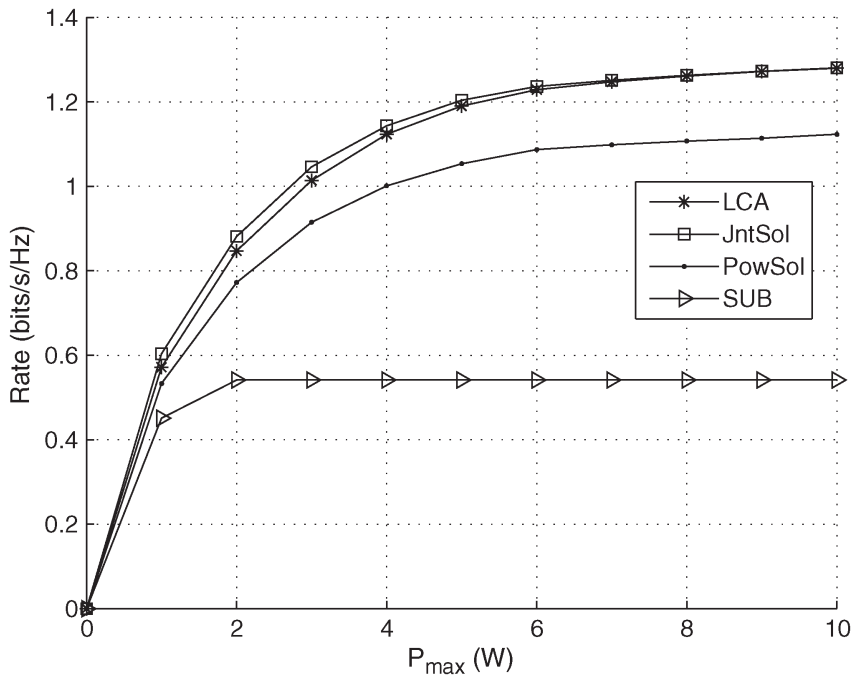

Fig. 6. Rate versus $P_{\max }$. Optimization under the per-subcarrier interference constraint and the per-node power constraint.

to the previous example, JntSol yields the best performance among the different methods, and the proposed suboptimal method (LCA) outperforms the solutions without subcarrier pairing (PowSol, SUB). Comparing the results with Fig. 5, all of the proposed schemes undergo a small performance loss at all $P_{\max }$ values, which is due to the strict protection criteria imposed by the PU. On the other hand, for the SUB method, we notice significant performance degradation in the high- $P_{\max }$ regime. Thus, the proposed optimization schemes are more resistive to the per-subcarrier interference threshold.

It is then of interest to check the impact of the interference threshold on the performance of the proposed methods. We consider optimization problem P1 and display the throughput curves versus $I_{\text {th }}$ at $P_{\max }=2$ in Fig. 7. The key observation is that the gap between JntSol and SUB first increases with an increasing interference threshold and finally becomes constant for high values of $I_{\mathrm{th}}$. This is due to the fact that in the high- $I_{\mathrm{th}}$ region, the system performance is restricted by power constraint (7) and becomes insensitive to $I_{\mathrm{th}}$. Other observations are similar to those in Fig. 5.

To obtain more insight on the impact of subcarrier pairing, in this example, we compare JntSol with PowSol and SUB for the following four cases.

Case 1: This is the same as in Fig. 5.

Case 2: $\left|h_{1}\right|^{2}>\left|h_{2}\right|^{2} \ldots>\left|h_{K}\right|^{2}$ and $\left|g_{1}\right|^{2}<\left|g_{2}\right|^{2} \ldots<\left|g_{K}\right|^{2}$. Case 3: $\left|h_{1}\right|^{2}>\left|h_{2}\right|^{2} \ldots>\left|h_{K}\right|^{2},\left|\widetilde{h}_{1}\right|^{2}>\left|\widetilde{h}_{2}\right|^{2} \ldots>\left|\widetilde{h}_{K}\right|^{2}$, $\left|g_{1}\right|^{2}>\left|g_{2}\right|^{2} \ldots>\left|g_{K}\right|^{2}$, and $\left|\widetilde{g}_{1}\right|^{2}<\left|\widetilde{g}_{2}\right|^{2} \ldots<\left|\widetilde{g}_{K}\right|^{2}$.

Case 4: $\left|h_{1}\right|^{2}>\left|h_{2}\right|^{2} \ldots>\left|h_{K}\right|^{2},\left|\widetilde{h}_{1}\right|^{2}<\left|\widetilde{h}_{2}\right|^{2} \ldots<\left|\widetilde{h}_{K}\right|^{2}$, $\left|g_{1}\right|^{2}<\left|g_{2}\right|^{2} \ldots<\left|g_{K}\right|^{2}$, and $\left|\widetilde{g}_{1}\right|^{2}>\left|\widetilde{g}_{2}\right|^{2} \ldots>\left|\widetilde{g}_{K}\right|^{2}$. 


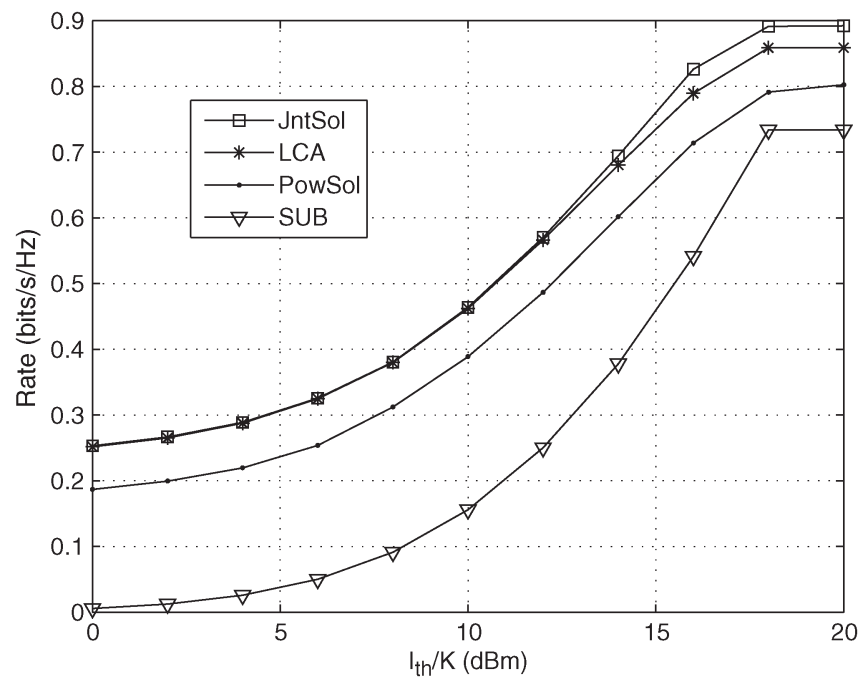

Fig. 7. Rate versus $I_{\mathrm{th}}$. Optimization under the sum interference constraint and the per-node power constraint.

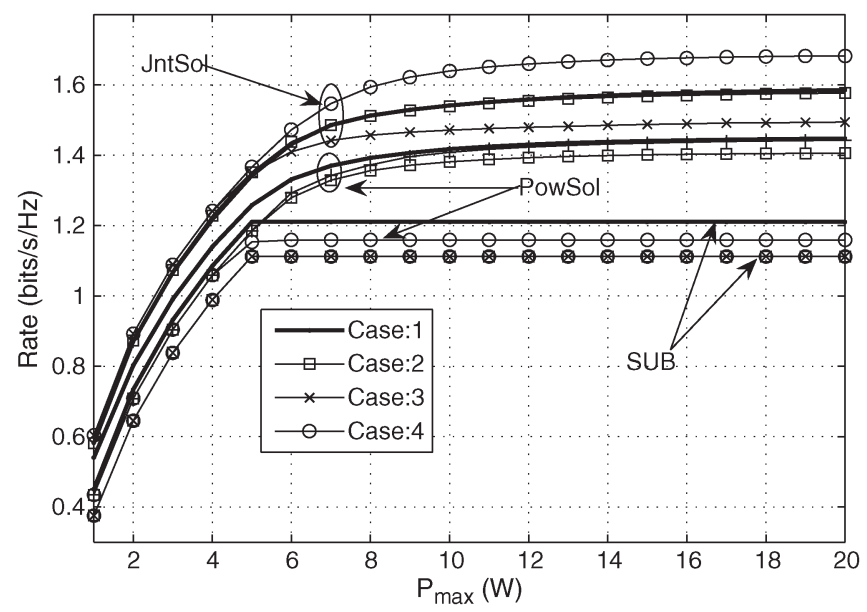

Fig. 8. Rate versus $P_{\max }$. Optimization under the sum interference constraint and the per-node power constraint.

The throughput versus $P_{\max }$ for the four cases at $I_{\mathrm{th}} / K=$ 0.15 is shown in Fig. 8. It can be seen that in Case 2, JntSol exhibits almost the same performance as in Case 1, where PowSol and SUB experience performance loss. This is because in PowSol and SUB algorithms, the stronger carrier at the first hop will always be matched to the weaker carrier over the second hop. For Case 3, all of the schemes (JntSol, PowSol, and SUB) undergo rate loss as compared with Case 1. The PowSol and SUB algorithms observe loss for a similar reason as in Case 2, whereas JntSol experiences performance degradation due to the fact that a subcarrier with the highest interference gain is always paired with a subcarrier of the highest interference. Nevertheless, JntSol still exhibits the best performance over the others. In Case 4, we first observe that the solution with equal power allocation is the same as in Case 2 and Case 3. However, PowSol and JntSol exhibit the most interesting behavior, i.e., PowSol experiences significant performance degradation, and JntSol yields much better performance compared with all the other cases. The gain in JntSol is due to the fact that the strongest subcarrier (with the smallest interference) is paired with the

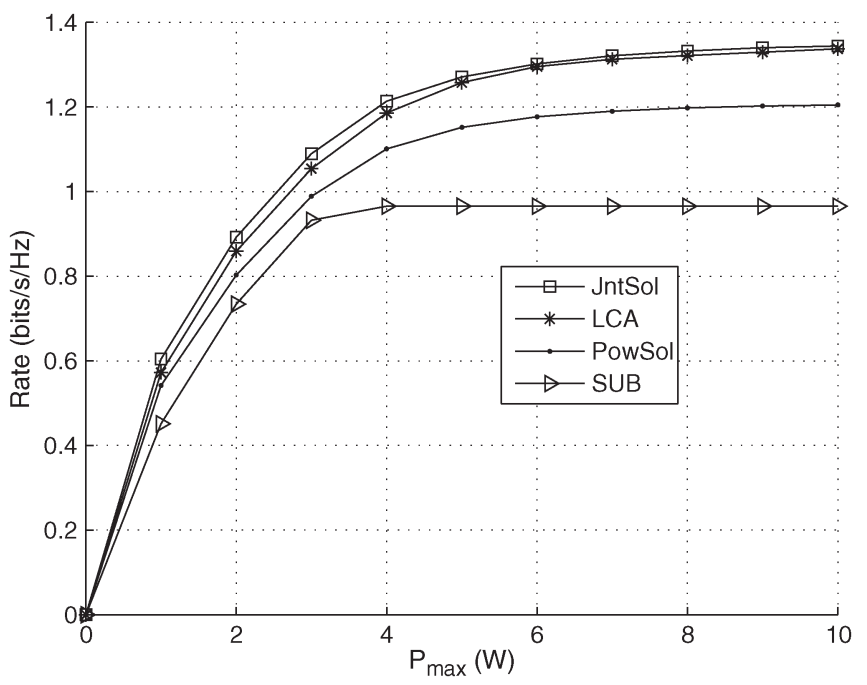

Fig. 9. Rate versus $P_{\max }$. Optimization under the sum interference constraint and the aggregate power constraint.

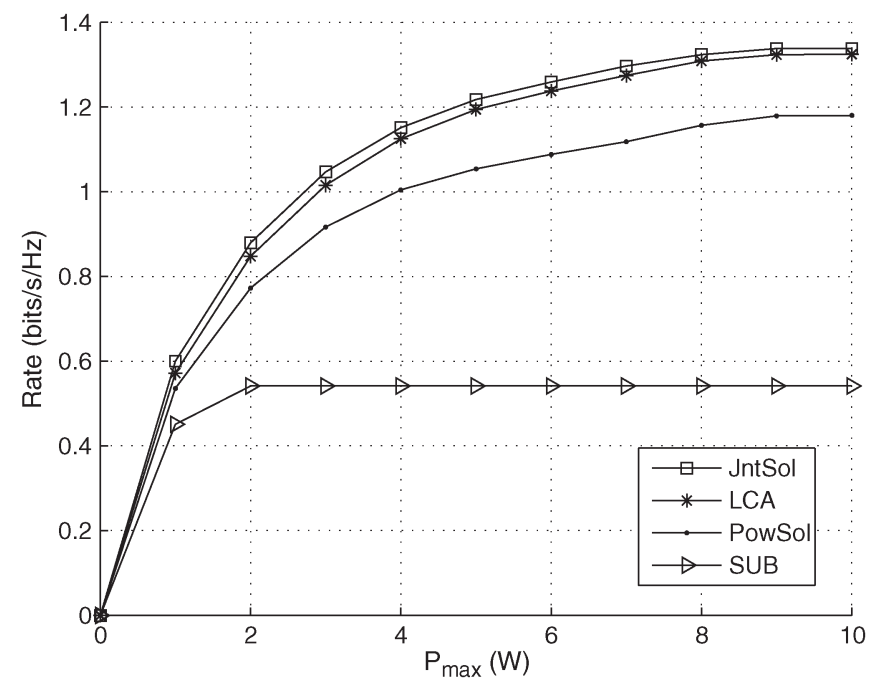

Fig. 10. Rate versus $P_{\max }$. Optimization under the per-subcarrier interference constraint and the aggregate power constraint.

strongest subcarrier (with the weakest interference), whereas PowSol suffers from the loss because a stronger subcarrier (with low interference) is paired with a weaker subcarrier (with higher interference).

Finally, we check the performance of the different schemes under the sum power constraint (8). The results with sum interference and per-subcarrier interference constraints are shown in Figs. 9 and 10, respectively. For comparison, we assume $P_{T}=P_{\mathrm{SSN}}+P_{\mathrm{SRN}}=2 P_{\max }$ and set $I_{\mathrm{th}}^{k}=I_{\mathrm{th}}^{j}=0.1, \forall k, j$. Similar to the previous examples, the proposed schemes yield significant gain over the trivial ones. Further, better performance is observed as compared with the results in Figs. 5 and 6 . This is because the sum power constraint provides more flexibility in power allocation over the two hops. In Figs. 5, 6,9 , and 10 , we observe that the throughput increases with $P_{\max }$ and becomes flat in the high- $P_{\max }$ region due to the interference constraints of the PU. It is further seen that in the SUB algorithm, the interference constraint becomes active 
at low $P_{\max }$ values as compared with the optimized powerallocation schemes and results in a large performance gap.

In Figs. 2-4, we can see that the number of iterations required for the convergence of the joint resource-allocation algorithm and the suboptimal algorithm is almost equal. However, the number of computations under each iteration is much greater in the joint optimization solution, as shown in Algorithm 1 and Algorithm 2. Furthermore, the suboptimal algorithm requires much less running time as compared with JntSol, as shown in Table I. On the other hand, in Figs. 5, 6, 9, and 10, we notice that the proposed suboptimal algorithm sacrifices very little on performance in comparison to the joint solution and yields significant gain over the trivial solutions. Further, similar behavior can be observed in Fig. 7, which shows the robustness of the suboptimal algorithm toward interference temperature. Thus, we conclude that the proposed suboptimal algorithm enjoys significant less computational complexity for a very small performance loss over the joint optimization solution.

\section{CONCLUSION}

In this paper, we have investigated the resource allocation problem in relay-aided OFDM CR networks. The SU's throughput is maximized via joint subcarrier pairing and power allocation. Meanwhile, the interference from both the secondary source and the SRN to the primary receiver is kept within an acceptable limit. A suboptimal resource-allocation algorithm was also designed to reduce computational complexity. The numerical examples demonstrated enhanced performance compared with trivial resource-allocation algorithms.

\section{APPENDIX}

The Lagrangian $J$ associated with (17) is

$$
\begin{aligned}
J=c_{(k, j)}-p_{k}\left(\lambda+\tilde{\lambda}\left|\tilde{h}_{k}\right|^{2}\right)- & q_{j}\left(\mu+\tilde{\mu}\left|\tilde{g}_{j}\right|^{2}\right) \\
& +\sum_{k=1}^{K} \eta_{k} p_{k}+\sum_{j=1}^{K} \gamma_{j} q_{j}
\end{aligned}
$$

where $\eta_{k}$ and $\gamma_{j}$ are the Lagrange multipliers.

From KKT condition $\partial J / \partial p_{k}=0$, we obtain

$$
\eta_{k}=\left(\lambda+\tilde{\lambda}\left|\tilde{h}_{k}\right|^{2}\right)-\frac{a_{k} q_{j}^{2} b_{j}^{2}}{\left(t_{(k, j)}\right)\left(t_{(k, j)}+a_{k} p_{k} b_{j} q_{j}\right)}
$$

where $t_{k, j} \triangleq a_{k} p_{k}+b_{j} q_{j}$. From KKT condition $\eta_{k} \geq 0$, we obtain

$$
\left(\lambda+\tilde{\lambda}\left|\tilde{h}_{k}\right|^{2}\right) \geq \frac{a_{k} q_{j}^{2} b_{j}^{2}}{\left(t_{(k, j)}\right)\left(t_{(k, j)}+a_{k} p_{k} b_{j} q_{j}\right)} .
$$

With the slackness condition $\alpha_{m, k} p_{m, k}=0$, we further arrive at

$$
p_{k}\left(\left(\lambda+\tilde{\lambda}\left|\tilde{h}_{k}\right|^{2}\right)-\frac{a_{k} q_{j}^{2} b_{j}^{2}}{\left(t_{(k, j)}\right)\left(t_{(k, j)}+a_{k} p_{k} b_{j} q_{j}\right)}\right)=0 .
$$

The following two cases hold.

a) If $\left(\lambda+\tilde{\lambda}\left|\tilde{h}_{k}\right|^{2}\right)<a_{k}$, then (34) can only be fulfilled when $p_{k}>0$. In this case, (35) holds only if

$$
\left(\lambda+\tilde{\lambda}\left|\tilde{h}_{k}\right|^{2}\right)=\frac{a_{k} q_{j}^{2} b_{j}^{2}}{\left(t_{(k, j)}\right)\left(t_{(k, j)}+a_{k} p_{k} b_{j} q_{j}\right)} .
$$

b) If $\left(\lambda+\tilde{\lambda}\left|\tilde{h}_{k}\right|^{2}\right) \geq a_{k}$, with $p_{k}>0$, it is impossible to meet (35). This implies that $p_{k}=0$.

Due to symmetry, for $q_{j}>0$, we obtain

$$
\left(\mu+\tilde{\mu}\left|\tilde{g}_{j}\right|^{2}\right)=\frac{b_{j} p_{k}^{2} a_{k}^{2}}{\left(t_{(k, j)}\right)\left(t_{(k, j)}+a_{k} p_{k} b_{j} q_{j}\right)} .
$$

Otherwise, $q_{j}=0$.

From (36) and (37), we conclude that for a particular subcarrier pair $(k, j)$, power values $p_{k}$ and $q_{j}$ are either both positive or both zero, i.e., if power allocated to a particular subcarrier over either hop is zero, no power is allocated to its corresponding subcarrier over the other hop, which meets the intuition very well. For $p_{m, k}>0$ and $q_{j}>0$, we can solve (36) and (37), which results in (18) and (19).

\section{REFERENCES}

[1] J. Miltola and G. Q. Maguire, "Cognitive radio: Making software radios more personal," IEEE Pers. Commun., vol. 6, no. 4, pp. 13-18, Aug. 1999.

[2] S. Force, "Spectrum policy task force report," Fed. Commun. Comm., Washington, DC, USA, ET Decket 02, Nov. 2002, vol. 135.

[3] S. Haykin, "Cognitive radio: Brain-empowered wireless communications," IEEE J. Sel. Areas Commun., vol. 23, no. 2, pp. 201-220, Feb. 2005.

[4] A. Goldsmith, S. A. Jafar, I. Maric, and S. Srinivasa, "Breaking spectrum gridlock with cognitive radios: An information theoretic perspective," Proc. IEEE, vol. 97, no. 5, pp. 894-914, May 2009.

[5] Q. Zhao and B. M. Sadler, "A survey of dynamic spectrum access," IEEE Signal Process. Mag., vol. 24, no. 3, pp. 79-89, May 2007.

[6] I. Hammerstrom and A. Wittneben, "On the optimal power allocation for nonregenerative OFDM relay links," in Proc. IEEE ICC, Jun. 2006, pp. 4463-4468.

[7] G. Bansal, M. J. Hossain, and V. K. Bhargava, "Adaptive power loading for OFDM-based cognitive radio systems," in Proc. IEEE ICC, Glasgow, U.K., Jun. 2007, pp. 5137-5142.

[8] S. Yan and X. Wang, "Power allocation for cognitive radio systems based on nonregenerative OFDM relay transmission," in Proc. IEEE Int. Conf. WiCom, Beijing, China, Sep. 2009, pp. 1-4.

[9] K. Son, B. C. Jung, S. Chong, and D. K. Sung, "Opportunistic underlay transmission in multi-carrier cognitive radio systems," in Proc. IEEE Int. Conf. WCNC, Budapast, Hungary, Apr. 2009, pp. 1-6.

[10] M. K. Shamim, M. K. Ahmed, and R. A. Shah, "Power allocation in OFDM-based cognitive relay networks," in Proc. IEEE Int. Conf. WCNIS, Beijing, China, Jun. 2010, pp. 202-206.

[11] J. Jia, J. Zhang, and Q. Zhang, "Cooperative relay for cognitive radio networks," in Proc. IEEE Int. Conf. INFOCOM, Rio de Janeiro, Brazil, Apr. 2009, pp. 2304-2312.

[12] H. J. Lim, D. Y. Seol, and G. H. Im, "Joint sensing adaptation and resource allocation for cognitive radio with imperfect sensing," IEEE Trans. Commun., vol. 60, no. 4, pp. 1091-1100, Apr. 2012.

[13] M. Naeem, U. Pareek, and D. C. Lee, "Power allocation for nonregenerative relaying in cognitive radio systems," in Proc. IEEE Int. Conf. WiMob, Niagara Falls, ON, Canada, Oct. 2010, pp. 720-725.

[14] L. Li, X. Zhou, H. Xu, G. Y. Li, D. Wang, and A. Soong, "Simplified relay selection and power allocation in cooperative cognitive radio systems," IEEE Trans. Wireless Commun., vol. 10, no. 1, pp. 33-36, Jan. 2011. 
[15] Q. Lu, T. Peng, W. Wang, W. Wang, and C. Hu, "Optimal route selection and resource allocation in multi-hop cognitive radio networks," in Proc. IEEE GLOBECOM, Honolulu, HI, USA, Dec. 2009, pp. 1-6.

[16] M. Herdin, "A chunk based OFDM amplify-and-forward relaying scheme for 4G mobile radio systems," in Proc. IEEE ICC, Istanbul, Turkey, Jun. 2006, pp. 4507-4512.

[17] I. Hammerstrom and A. Wittneben, "Power allocation schemes for amplify-and-forward MIMO-OFDM relay links," IEEE Trans. Wireless Commun., vol. 6, no. 8, pp. 2798-2802, Aug. 2007.

[18] Y. Li, W. Wang, J. Kong, and M. Peng, "Subcarrier pairing for amplifyand-forward and decode-and-forward OFDM relay links," IEEE Commun. Lett., vol. 13, no. 4, pp. 209-211, Apr. 2009.

[19] G. A. S. Sidhu, F. Gao, W. Chen, and A. Nallanathan, "A joint resource allocation scheme for multiuser two-way relay networks," IEEE Trans. Commun., vol. 59, no. 11, pp. 2970-2975, Nov. 2011.

[20] F. Gao, R. Zhang, and Y.-C. Liang, "Channel estimation for OFDM modulated two-way relay networks," IEEE Trans. Signal Process., vol. 57, no. 11, pp. 4443-4455, Nov. 2009.

[21] F. Gao, B. Jiang, X.-Q. Gao, and X.-D. Zhang, "Superimposed training based channel estimation for OFDM modulated amplify-and-forward relay networks," IEEE Trans. Commun., vol. 59, no. 7, pp. 2029-2039, Jul. 2011.

[22] D. J. Love, R. W. Heath, V. K. N. Lau, D. Gesbert, B. D. Rao, and M. Andrews, "An overview of limited feedback in wireless communication systems," IEEE J. Sel. Areas Commun., vol. 26, no. 8, pp. 1341-1365, Oct. 2008

[23] K. Huang and R. Zhang, "Cooperative feedback for multiantenna cognitive radio networks," IEEE Trans. Signal Process., vol. 59, no. 2, pp. 747758, Feb. 2011.

[24] T. Qin, C. Leung, C. Miao, and Z. Shen, "Resource allocation in a cognitive radio system with imperfect channel state estimation," J. Elect. Comput. Eng., vol. 2010, pp. 419430-2-419430-5, Jan. 2010.

[25] J. Tang and X. Zhang, "Cross-layer resource allocation over wireless relay networks for quality of service provisioning," IEEE J. Sel. Areas Commun., vol. 25, no. 4, pp. 645-656, May 2007.

[26] W. Yu and R. Lui, "Dual methods for nonconvex spectrum optimization of multicarrier systems," IEEE Trans. Commun., vol. 54, no. 7, pp. 13101322, Jul. 2006.

[27] Z. Luo and S. Zhang, "Dynamic spectrum management: Complexity and duality," IEEE J. Sel. Topics Signal Process., vol. 2, no. 1, pp. 57-73, Feb. 2008.

[28] S. Boyd and L. Vandenberghe, Convex Optimization. Cambridge, U.K. Cambridge Univ. Press, 2004.

[29] H. Khun, "The Hungarian method for the assignment problems," Naval Res. Logist. Quart., vol. 2, no. 1/2, pp. 83-97, Mar. 1955.

[30] N. Z. Shor, Minimization Methods for Non-Differentiable Functions. New York, NY, USA: Springer-Verlag, 1985, Springer Series in Computational Mathematics.

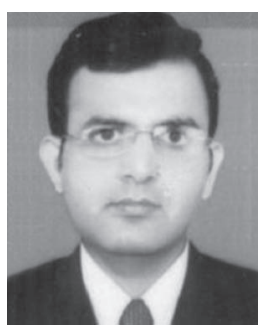

Guftaar Ahmad Sardar Sidhu received the B.Sc. degree in computer engineering from COMSATS Institute of Information Technology, Islamabad, Pakistan, in 2006 and the M.Sc. and Ph.D. degrees in electrical engineering from Jacobs University, Bremen, Germany, in 2009 and 2012, respectively.

$\mathrm{He}$ is currently an Assistant Professor with the Department of Electrical Engineering, COMSATS Institute of Information Technology. He has published his work in international journals and peerreviewed conferences. His research interests include cooperative communication, cognitive radio networks, wireless sensor networks, and multiuser orthogonal frequency-division multiplexing systems.

Dr. Sidhu served as a Technical Program Committee Member for the 23rd IEEE International Symposium on Personal, Indoor, and Mobile Radio Communications and a Reviewer for the IEEE TRANSACTIONS ON COMMUNICATIONS, the IEEE TRANSACTIONS ON VEHICULAR TECHNOLOGY, the IEEE COMMUNICATIONS LETTERS, the IEEE WIRELESS COMMUNICATION LETTERS, the IEEE International Conference on Communications, and the IEEE Global Communications Conference (GLOBECOM). He received the Best Paper Award at the IEEE International Wireless Communication and Signal Processing Conference in 2010.

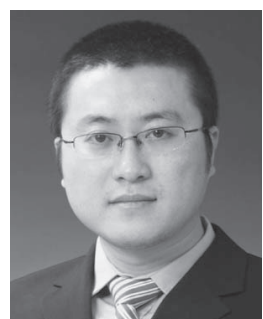

Feifei Gao (S'05-M'09) received the Ph.D. degree from National University of Singapore, Singapore, in 2007

In 2008, he was a Research Fellow with the Institute for Infocomm Research, A*STAR, Singapore. From 2009 to 2010, he was an Assistant Professor with the School of Engineering and Science, Jacobs University, Bremen, Germany. In 2011, he joined the Department of Automation, Tsinghua University, Beijing, China, where he is currently an Associate Professor. He is also an Adjunct Professor with the School of Engineering and Science, Jacobs University. He has authored and coauthored more than 50 refereed IEEE journals and more than 70 IEEE conference proceeding papers.

Dr. Gao has served as an Editor for the IEEE TRANSACTIONS ON WIRELESS COMMUNICATIONS and the IEEE WIRELESS COMMUNICATIONS LETTERS.

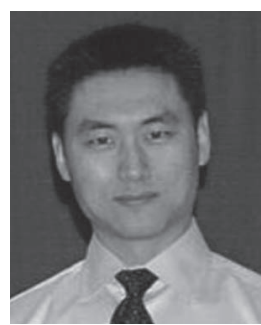

Wei Wang received the Ph.D. degree in computer engineering from the University of NebraskaLincoln, NE, USA, in 2009.

$\mathrm{He}$ is an Assistant Professor with the Department of Electrical Engineering and Computer Science, South Dakota State University, Brookings, SD, USA He has published over 70 research articles. His research interests include wireless sensor networks, multimedia communications, information security, and educational robotics.

Dr. Wang served as an Associate Editor for the Wiley Security and Communication Networks Journal, the Workshop Cochair of the Institute for Computer Sciences, Social Informatics and Telecommunications Engineering Conference on Body Area Networks (ICST BodyNet2013), the Track Chair of the IEEE International Conference on Computer and Information Technology (CIT2012-MMC), the Vice Chair of the IEEE International Conference on Communication Technology (ICCT2011-NGN) track, and the Program Chair of the ICST International Workshop on Mobile Multimedia Networking (IWMMN2010). He has also served as a Technical Program Committee Member of the IEEE Global Communications Conference (GLOBE$\mathrm{COM}$ ), the IEEE International Conference on Communications, and the IEEE Wireless Communications and Networking Conference (WCNC). He received two Best Paper Awards at the IEEE WCNC in 2008 and the Annual Simulation Symposium in 2011.

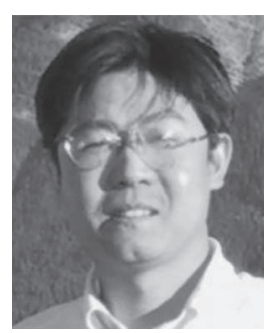

Wen Chen (M'03-SM'11) received the B.S. and M.S. degrees from Wuhan University, Wuhan, China, in 1990 and 1993, respectively, and the Ph.D. degree from the University of Electro-Communications, Tokyo, Japan, in 1999.

From 1999 to 2001, he was a Researcher with the Japan Society for the Promotion of Sciences. In 2001, he joined the University of Alberta, Edmonton, $\mathrm{AB}$, Canada, starting as a Postdoctoral Fellow with the Information Research Laboratory and continuing as a Research Associate with the Department of Electrical and Computer Engineering. Since 2006, he has been a Full Professor with the Department of Electronic Engineering, Shanghai Jiao Tong University, Shanghai, China, where he is also the Director of the Institute for Signal Processing and Systems. In 2008, he was elected as the Vice General Secretary of the Shanghai Institute of Electronics. He has published more than 100 papers in IEEE journals and conferences. His interests include network coding, cooperative communications, cognitive radio, and multiple-input-multiple-output orthogonal frequency-division multiplexing systems.

Dr. Chen received the Ariyama Memorial Research Prize in 1997 and the Pacific Institute for the Mathematical Sciences Postdoctoral Fellowship in 2001. He was a New Century Excellent Scholar in China in 2006 and a Pujiang Excellent Scholar in Shanghai in 2007. He is in the editorial board of the International Journal of Wireless Communications and Networking and serves as a Lead Guest Editor for the Journal of Communications, Journal of Computers, Journal of Networks, and the European Association for Signal Processing (EURASIP) Journal on Wireless Communications and Networking. He was the Technical Program Committee Chair for the IEEE International Conference on Circuits and Systems for Communications (ICCSC2008) and the 14th IEEE International Conference on Communication Technology (ICCT2012) and the General Conference Chair for the IEEE International Conference on Intelligent Computing and Intelligent Systems (ICIS2009); the IEEE International Conference on Wireless Communications, Networking, and Information Security (WCNIS2010); and the IEEE International Conference on Wireless and Mobile Computing, Networking, and Communications (WiMob2011). 